\title{
More options for gene editing
}

Engineered nucleases work across species and in isogenic stem cells.

Engineering precise genetic changes in a genome is powerful way to study gene function, and several recent papers describe new applications of geneediting tools. Working with researchers at Sangamo BioSciences, Howard Hughes Medical Institute investigator Barbara Meyer and her colleagues at the University of California, Berkeley, described the first systems for making targeted genomic modifications in the roundworm Caenorhabditis elegans, a valuable model organism (Wood et al., 2011).

Following previously established techniques, the researchers created geneediting complexes using fusion proteins. They paired the same nuclease with one of two types of DNA-recognition units: zinc-finger motifs or truncated transcription activator-like effector domains. The team found they could overcome previous difficulties in introducing gene-editing complexes into cells by injecting mRNA encoding them into worm gonads. Not only did these complexes efficiently introduce desired changes into the genome, they also showed very little off-target editing. In contrast to knockdown technologies such as RNA interference, these gene-editing complexes produce stable and heritable mutations, says Meyer. "We can do reverse genetics in an animal for which no other genetic approaches have been established," she says.

The researchers went on to show that zinc finger nucleases (ZFNs) also worked in a second roundworm, C. briggsae, which diverged from C. elegans 15 to $30 \mathrm{mil}$ lion years ago. Being able to probe DNA sequences across species will allow new studies about how evolution happens, says Meyer. For example, different species of roundworms have similar protein machinery that recognizes very divergent DNA sequences. The ability to edit these sequences will allow researchers to explore how DNA-recognition elements evolve and diverge.

Whereas exploring the same mutation in different species can be useful, so can exploring specific genetic variants in otherwise identical cells. Researchers have generated induced pluripotent stem (iPS) cells from many individuals with diseases including Alzheimer's disease, heart disease and diabetes. But mutations that contribute to such conditions will likely have subtle phenotypes in cultured cells. That makes distinguishing relevant changes from background variation difficult.

To get around this problem, researchers led by Rudolf Jaenisch at the Whitehead Institute generated iPS cells from individuals with and without early onset Parkinson's disease. Next, they used ZFNs to alter the a-synuclein gene, creating isogenic lines differing exclusively at known susceptibility variants (Soldner et al., 2011).

In a separate paper, Jaenisch and colleagues showed that transcription activator-like effector nucleases (TALENs) could also be used to edit genomes of human iPS cells (Hockemeyer et al., 2011). The team generated TALENs for each of five genomic loci and edited DNA sequences at each site. Overall, the researchers concluded, TALENs had similar precision and efficiency of genome editing as ZFNs.

Meanwhile, the utility of ZFNs continues to expand. They have already been used in human cells, mouse, rat and zebrafish. Researchers led by Heiner Niemann at the Institute of Farm Animal Genetics in Germany in collaboration with Sangamo scientists have now used the enzymes to make genetically modified pigs, showing that the technology could be useful for cell and organ transplants (Hauschild et al., 2011).

ZFNs have been more thoroughly studied than the more recently discovered TALENs, says Meyer, but in her experience TALENs are easier to design and to validate as working with a desired

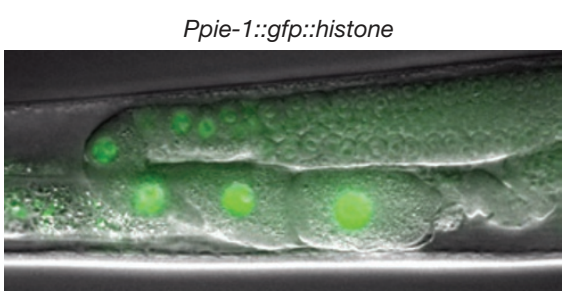

Ppie-1::gfp::histone mutation

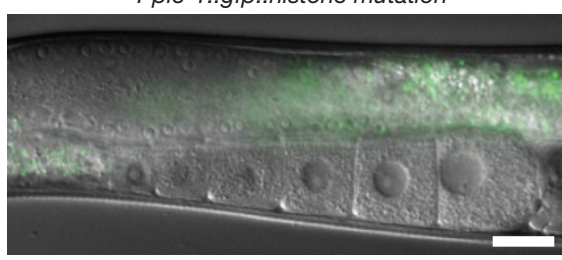

DNA editing by ZFNs disrupts a reporter gene in a nematode. Scale bar, 20 micrometers. Image courtesy of B. Meyer.

sequence. But Philip Gregory, chief scientific officer of Sangamo BioSciences, says that ZFNs are still the preferred editing tool because researchers have not yet collected enough evidence to understand how often TALENs produce off-target effects.

Sangamo has already commercialized ZFNs (these are being developed and distributed as research reagents with Sigma-Aldrich) and is continuing work on TALENs. Both ZFNs and TALENs will continue to become more useful, says Gregory. "The more tools the field has, the more options we have to create genomes that are edited in precisely the right way."

\section{Monya Baker}

\section{RESEARCH PAPERS}

Hauschild J. et al. Efficient generation of a biallelic knockout in pigs using zinc-finger nucleases. Proc. Natl. Acad. Sci USA 108, 12013-12017 (2011). Hockemeyer, D. et al. Genetic engineering of human pluripotent cells using TALE nucleases. Nat. Biotechnol. 29, 731-743 (2011).

Soldner, F. et al. Generation of isogenic pluripotent stem cells differing exclusively at two early onset Parkinson point mutations. Cell 146, 318-331 (2011).

Wood, A. J. et al. Targeted genome editing across species using ZFNs and TALENs. Science 333, 307 (2011). 